\title{
Editorial Referateband 2018
}
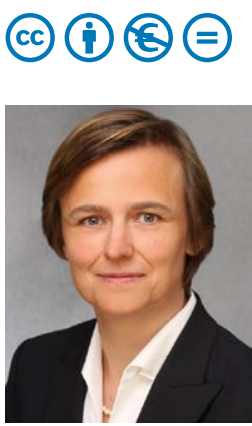

Prof. Dr. med. Barbara Wollenberg

\author{
Korrespondenzadresse \\ Prof. Dr. med. Barbara Wollenberg \\ Univ. HNO-Klinik \\ Ratzeburger Allee 160 \\ D-23538 Lübeck \\ Tel.: + 49/451500 42001, Fax: +49/45150042004 \\ Bibliografie \\ DOI https://doi.org/10.1055/s-0044-101376 \\ Laryngo-Rhino-Otol 2018; 97: S1 \\ (c) Georg Thieme Verlag KG Stuttgart · New York \\ ISSN 1615-0007
}

Liebe Kolleginnen und Kollegen,

mit großer Freude lege ich den Referateband zur 89. Jahresversammlung der Deutschen Gesellschaft für Hals-Nasen-Ohren-Heilkunde, Kopf- und Hals-Chirurgie vor. Dem Kongressmotto „Forschung heute - Zukunft morgen " folgend werden einige der Themen im Detail beleuchtet, die in den nächsten Jahren das Potential haben, die Versorgung unserer Patienten grundlegend zu verändern.

Es sind Themen aus ganz unterschiedlichen grundlagenwissenschaftlichen Fachbereichen, die sich in vielen klinischen Bereichen einsetzen lassen.

Ein weiterhin großes Problem stellt die unverändert limitierte Überlebenschance der Patienten mit Kopf-Halskarzinom dar. Trotz der Erweiterung der Chirurgie um diverse rekonstruktive Verfahren, wie auch die Erweiterung der strahlentherapeutischen Maßnahmen, bleibt die Prognose und Überlebensrate der Patienten in den fortgeschrittenen Erkrankungsstadien schlecht. Daher greifen die Referate die Entwicklungen im Bereich der Onkologie auf. Es geht um die Charakterisierung der „zweiten Entität des Kopf-HalsKarzinoms“, das durch HPV Viren initiiert, sich molekular, biologisch, immunologisch und damit wahrscheinlich in Zukunft auch therapeutisch vom klassischen Noxen induziertem Kopf-Halskarzinom unterscheidet. Hierbei kommt das hochaktuelle Gebiet der Immuntherapie gerade zum richtigen Zeitpunkt. Durch eine Vielzahl von Antikörpern, die gerade ihren Übergang aus der Entwicklung in die klinische Routine finden, gelingt es, die Zellen der körpereigenen Immunantwort gegen Krebszellen zu aktivieren. Die aktuelle klinische Datenlage weist für bestimmte Patienten einen merklichen Überlebensvorteil aus. Auch die diagnostischen Verfahren der Onkologie ändern sich, weniger invasiv, auf „Big Data“ gestützt, werden verlässliche prognostische Indikatoren an Schnittbildern abgeleitet, die klinische Verdachtsmomente hilfreich unterstützen können. Die Verknüpfung von Bildgebung und Technik spielt auch bei der robotischen Chirurgie eine relevante Rolle. Die Darstellung der aktuellen Systeme, Vorteile, aber auch Limitationen werden ausführlichen Raum bekommen.

An der Schnittstelle von Onkologie zur Rhinologie steht das Mikrobiom. Seit wenigen Jahren wird offensichtlich, dass der menschliche Körper von mehr Bakterien besiedelt ist, als es körpereigene Zellen gibt. Der Einfluss dieser Bakterien auf relevante, entzündungsbedingte Krankheitsbilder, wie Karzinome oder rhinologische Erkrankungen, steht an der Schwelle der Erforschung. Die Therapie der chronischen Rhinosinusitiden wechselt aus den chirurgischen Verfahren in die antikörpergestützte Therapie, wieder eine Art Immuntherapie. Nach einem so genannten „Entzündungs-Endotyping“ werden verschiedene individualisierte Antikörpertherapien entstehen.

Mit großen Schritten entwickelt sich auch das Verständnis des molekularen Hörvorganges. Diese Einsichten werden zukünftig nachhaltig die Versorgung der Patienten mit Schwerhörigkeit in der Prävention, Diagnostik und operativen Versorgung verändern.

Auch die regenerative Medizin spielt für die Zukunft der HNOHeilkunde eine große Rolle, durch neuartige Ansätze kommen erste Einsatzmöglichkeiten im klinischen Alltag an.

Die Vielseitigkeit der hier gebündelten innovativen Forschungsansätze zeigt, welchen Entwicklungsbedarf und auch -chancen die HNO-Heilkunde aufweist. Mögen die hier gesammelten Referate den Grundstein zu der Faszination an Forschung sein, die unser Fach weiter in die Zukunft trägt.

Viel Vergnügen mit den Referaten zu „Forschung heute Zukunft morgen“

Prof. Dr. med. Barbara Wollenberg

Präsidentin der Deutschen Gesellschaft für HNO-Heilkunde, Kopf- und Hals-Chirurgie 2017/2018 